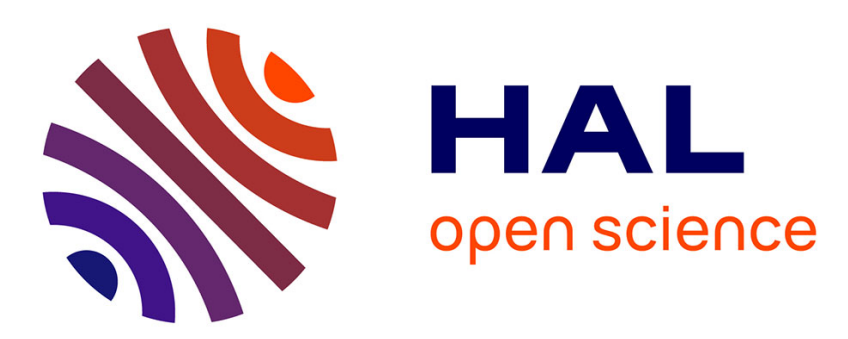

\title{
Détection de photoneutrons avec un scintillateur liquide chargé au gadolinium
}

H. Beil, R. Bergère, A. Veyssière

\section{To cite this version:}

H. Beil, R. Bergère, A. Veyssière. Détection de photoneutrons avec un scintillateur liquide chargé au gadolinium. Revue de Physique Appliquée, 1969, 4 (2), pp.249-250. 10.1051/rphysap:0196900402024900 . jpa-00243245

\section{HAL Id: jpa-00243245 https://hal.science/jpa-00243245}

Submitted on 1 Jan 1969

HAL is a multi-disciplinary open access archive for the deposit and dissemination of scientific research documents, whether they are published or not. The documents may come from teaching and research institutions in France or abroad, or from public or private research centers.
L'archive ouverte pluridisciplinaire HAL, est destinée au dépôt et à la diffusion de documents scientifiques de niveau recherche, publiés ou non, émanant des établissements d'enseignement et de recherche français ou étrangers, des laboratoires publics ou privés. 


\title{
DÉTEGTION DE PHOTONEUTRONS AVEG UN SGINTILLATEUR LIQUIDE GHARGÉ AU GADOLINIUM
}

\author{
H. BEIL, R. BERGËRE et A. VEYSSIÈRE, \\ Service des Mesures Neutroniques Fondamentales, C.E.N., Saclay.
}

\begin{abstract}
Résumé. - Un scintillateur liquide chargé au gadolinium $(0,5 \%$ en poids) et employé récemment à Saclay comme détecteur de photoneutrons est décrit. Des efficacités de détection de neutrons $\varepsilon$ de l'ordre de $85 \%$ peuvent être atteintes. Ceci a permis de séparer les interactions $(\gamma, 3 \mathrm{n})$ des interactions $(\gamma, 2 \mathrm{n})$ et $(\gamma, \mathrm{n})$. Nous indiquons également la bonne concordance entre les résultats expérimentaux et théoriques pour les probabilités de capture des neutrons par Gd, dans la boule. Le bruit de fond total a été réduit à $6 \times 10^{-3}$ coup par porte de $25 \mu \mathrm{s}$.

Abstract. - The essential characteristics of a large, gadolinium loaded $\left(0.5{ }^{\circ}\right.$ in weight), liquid scintillator for neutron detection, used in photonuclear work at Saclay, are described. Eifficiencies of the order of $85 \%$ are obtainable thus making observations of photonuclear reactions of the type $(\gamma, 3 \mathrm{n})$ or $(\gamma, 2 \mathrm{n})$ possible. Reasonable agreement between theoretical and experimental time histories for neutron capture inside the detector exists. Total background is reduced to $6 \times 10^{-3}$ count per $25 \mu$ s time gate.
\end{abstract}

I. Introduction. - Nous présentons ici les caractéristiques principales d'un scintillateur liquide organique de grandes dimensions, chargé au gadolinium et employé récemment à Saclay comme compteur de neutrons dans une série d'expériences photonucléaires sur les terres rares [1]. Des détecteurs semblables furent introduits essentiellement pour des mesures de $\bar{v}$ [2-3]. Les avantages principaux d'un tel détecteur sont d'abord sa grande efficacité de détection des neutrons $\varepsilon$ et le fait que $\varepsilon$ est pratiquement indépendant de l'énergie des neutrons pour $E_{\mathrm{n}} \leqslant 5 \mathrm{MeV}$ [4]. La grande efficacité $(\varepsilon \not 85 \%)$ a permis de séparer les interactions $(\gamma, 3 \mathrm{n})$ des interactions $(\gamma, 2 \mathrm{n})$ et $(\gamma, \mathrm{n})$. Le désavantage principal est la sensibilité du scintillateur aux différentes sources de bruits de fond. Le principe de fonctionnement est le suivant : un neutron injecté au centre de la sphère est ralenti par choc élastique avec le liquide donnant ainsi naissance à une impulsion « prompte » due au premier proton de recul. Ensuite, le neutron est thermalisé et capturé par Gd. Les $\gamma$ de capture produisent des scintillations dont la somme donne une impulsion « capture de neutrons ». Une étude théorique approfondie sur de tels détecteurs [4] est en bon accord avec nos résultats expérimentaux.

II. Appareillage et méthodes expérimentales. La figure 1 montre le dispositif expérimental. Le blindage est conçu de façon que les neutrons parasites thermalisés puissent être absorbés dans Li. Le détecteur a été fabriqué par « Nuclear Enterprises » et contient 5001 de liquide scintillant du type NE 323 chargé au gadolinium $(0,5 \%$ en masse). La cible est placée

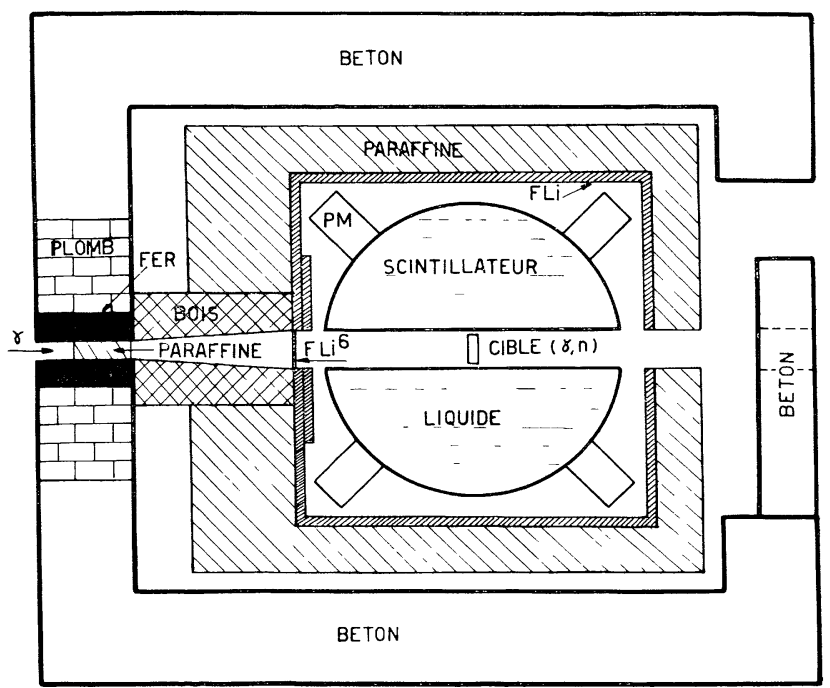

FIG. 1. - Dispositif expérimental : béton (lourd), $50 \mathrm{~cm}$; bois, $90 \mathrm{~cm}$; plomb et fer, $50 \mathrm{~cm}$; ( $\mathrm{FLi}+$ araldite) autour de la boule, de 2,5 à $5 \mathrm{~cm} ; \mathrm{F}^{6} \mathrm{Li}$ directement dans le faisceau, $0,5 \mathrm{~g} / \mathrm{cm}^{2}$; paraffine autour de la boule, de 80 à $40 \mathrm{~cm}$; paraffine dans le faisceau, $18,6 \mathrm{~g} / \mathrm{cm}^{2} ; \varnothing(\gamma), 4 \mathrm{~cm} ; \varnothing$ (tuyau central de la boule), $15 \mathrm{~cm}$.

au centre d'un cylindre de $\varnothing=15 \mathrm{~cm}$ et le faisceau de $\gamma$ monochromatiques collimaté à $\varnothing=4 \mathrm{~cm}$ est créé par la méthode d'annihilation en vol de positons [5]. La figure 2 donne le schéma synoptique de l'électronique associée. On y remarque que le «AEM » 


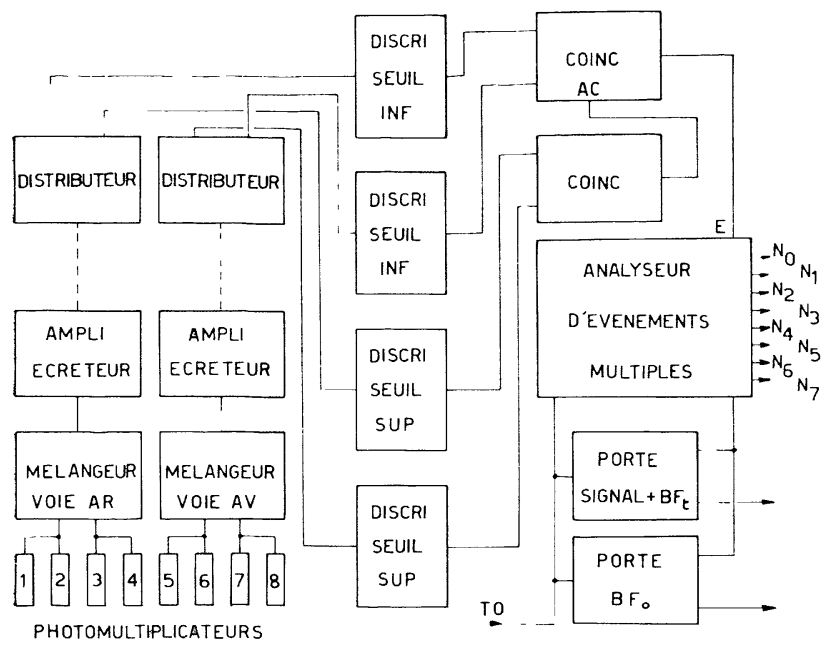

Fig̣. 2. - Schéma synoptique de l'électronique : L'analyseur d'événements multiples (A.E.M.) est un circuit logique capable de séparer et classifier les interactions photonucléaires en fonction du nombre de neutrons émis $(N=0$ à $N=7)$.

ne peut envoyer les données (multiplicité des interactions nucléaires, bruits de fond total $B F$ et ambiant $B F_{0}$, etc.) dans les différentes échelles $\mathrm{N}_{0}$ à $\mathrm{N}_{7}$, que par des portes électroniques à leur tour contrôlées par le temps zéro $\left(T_{0}\right)$. La figure 3 montre la séquence

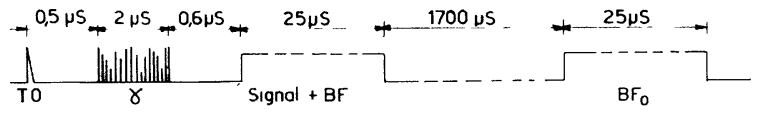

FIG. 3. - Représentation schématique des séquences et largeurs des portes en temps : $T_{0}$ représente le moment de démarrage d'un cycle d'enregistrement de données ; $B F$, bruit de fond total ; $B F_{0}$, bruit de fond ambiant.

et les largeurs des différentes portes. Pour les mesures de $\varepsilon$ ainsi que pour la distribution en temps de la capture des neutrons, $T_{\mathbf{0}}$ est donné par un fragment de fission provenant d'une source de ${ }^{252} \mathrm{Cf}$ placée au centre de la boule. On peut calculer, par des méthodes de Monte-Carlo [4], que 97,5\% des neutrons détectés seront ainsi comptés dans une porte de $35 \mu$ s après les protons de recul. Pour une mesure de $\varepsilon$, on aura donc (fig. 3) : $T_{0}=$ fission, puis un délai de $1,6 \mu$ s suivi par la première porte dans laquelle on observera les neutrons de fission plus $B F=B F_{0}$. La deuxième porte donnera $B F_{0}$ seulement. Pour les mesures photonucléaires, $T_{0}$ est donné par la fréquence de répétition $(500 \mathrm{~Hz})$ du linac de $45 \mathrm{MeV}$. Dans ce cas, les séquences et les intervalles de la figure 3 sont impératifs afin d'obtenir des rapports signal/bruit acceptables.

On peut écrire $B F=B F_{0}+B F Q+B F T$, où $B F Q$ est un bruit de fond, fonction du nombre et de l'énergie des charges $Q^{+}$ou $Q^{-}$arrivant sur la cible d'annihilation et $B F T$ un bruit de fond, fonction du courant dans le linac [6].

III. Résultats. - III. 1. BRUIT DE FOND (exprimé en nombre de coups par porte de $25 \mu \mathrm{s}): B F_{0}=4 \times 10^{-3}$; $\overline{B F T}=10^{-3} ; B F Q=f\left(E_{\gamma}, Q^{ \pm}\right) ; B F Q=0$ pour des énergies $E_{\gamma} \leqslant 13,3 \mathrm{MeV} ; B F Q \cong 10^{-3}$ pour $E_{\gamma}$ $=20 \mathrm{MeV}$. On obtient donc $B F \cong 6 \times 10^{-3}$ pour $E_{\gamma}=20 \mathrm{MeV}$.

III.2. Probabilité de Gapture des neutrons. Pour des neutrons provenant du centre de la sphère, on peut écrire en première approximation [4] que la probabilité de capture d'un neutron par le gadolinium entre les instants $T$ et $T+\mathrm{d} T$ est donnée par :

$$
\frac{\mathrm{d} h(T)}{\mathrm{d} T} \mathrm{~d} T=\mathrm{d} T\left\{\int_{0}^{T} \lambda^{2} t \mathrm{e}^{-\lambda t} \beta \mathrm{e}^{-\beta(T-t)} \mathrm{d} t\right\}
$$

où $\beta$ et $\lambda$ sont des paramètres dépendant des caractéristiques du détecteur. Avec cette formule, nous trouvons que les probabilités de capture maximale sont à $6 \mu$ s pour $0,5 \%$ de Gd et à $7 \mu$ s pour $0,3 \%$ de Gd. Ces résultats, ainsi que la forme théorique de la distribution de probabilités des captures ont été vérifiés par nos mesures. La distribution de probabilité du bruit ambiant $\left(\frac{\mathrm{d} h(T)}{\mathrm{d} T} \mathrm{~d} T\right)_{B F_{0}}$ est une constante; par contre, celle du bruit de fond accélérateur $B F T$ suit grosso modo la forme théorique avec un maximum vers $17 \mu \mathrm{s}$.

III.3. EfficaGité DE dÉtegtion DES NEUTRONS. Pour un spectre de neutrons de fission de ${ }^{252} \mathrm{Cf}$ $(\bar{\nu}=3,784)$, on peut obtenir une efficacité $\varepsilon$ de l'ordre de $85 \%$, mais la plupart des expériences ont été exécutées avec $\varepsilon=60 \%$ afin d'obtenir un meilleur rapport : signal/bruit de fond $=100 \times 10^{-3} / 6 \times 10^{-3}$ $\cong 16$. Le calcul montre que $\varepsilon$ (neutrons de $1 \mathrm{MeV}$ )/ $\varepsilon$ (neutrons de $5 \mathrm{MeV}$ ) $=98 / 90$, le détecteur pouvant ainsi être considéré comme ayant une efficacité à peu près indépendante de l'énergie dans la gamme d'énergie où nous travaillons.

\section{BIBLIOGRAPHIE}

[1] Bergère (R.) et al., Nucl. Phys., 1968, A 121, 463.

[2] Mather (D. S.), Fieldhouse (P.) et Moat (A.), Phys. Reu., 1964, 133, B 1403.

[3] Fréhaut (J.) et al., J. Physique, 1968, 29, C1-157.
[4] Parker (J. B.) et al., Nucl. Instr. Methods, 1968, 60, 7.

[5] Milier (J.), SchuHi, (C.) et Tzara (C.), Nucl. Phys., 1962, 32, 236.

[6] VEYSSIÈre (A.) et al. (en cours de publication). 